\title{
PHƯƠNG PHÁP XỬ LÝ SỐ LIỆ QUAN TRĂC LIÊN TỤC XÁC ĐỊNH THAM SỐ DAO ĐỘNG CÔNG TRİNH
}

\author{
PGS. TS. VŨ THĀNG \\ ThS. BÙI DUY QUYYNH \\ ThS. VŨ THÁl HÀ \\ Trường Đại học Xây dụng
}

\section{Tóm tắt:}

Bài báo giới thiệu kết quả nghiên cứu mô hình đo liên tục và phương pháp xử lý số liệu đo để xác định các tham số dao động công trình xây dựng dựa trên kết quả đo của các thiết bị hiện đại, có khả năng tự động đo liên tục như GPS, TĐĐT.

\section{Mở đầu}

Những công trình xây dựng đặc biệt do tác động của ngoại lực dẫn đến bị dao động, rung lắc làm ảnh hưởng tới công tác trắc địa cũng như an toàn trong quá trình thi công, độ ổn định trong thời gian khai thác sử dụng. Việc xác định các tham số dao động dựa trên kết quả đo của các thiết bị hiện đại, có khả năng tự động đo liên tục như GPS (Global Positioning System), TĐĐT (Toàn Đạc Điện Tử). Xử lý kết quả đo xác định các tham số dao động là nội dung bài báo đề cập

\section{Tham số dao động công trình}

Để xác định dao động, rung lắc công trình cần quan trắc sự thay đổi tuần hoàn vị trí những điểm đặc trưng trên công trình.

Dao động công trình có tính tuần hoàn, phương trình dao động có dạng [7],[8]:

$$
\mathrm{Q}_{\mathrm{t}}=\mathrm{a}_{0}+\mathrm{a}_{1} \cos (\omega \mathrm{t})+\mathrm{b}_{1} \sin (\omega \mathrm{t})
$$

trong đó :

$Q_{\mathrm{t}}$ là sự chuyển dịch của công trình.

t là thời điểm của dao động;

tần số góc $\omega=2 \pi f$

$a_{0}$ là độ dịch chuyển trung bình của đối tượng;

$a_{1}, b_{1}$ là các hệ số Fourier của hàm số.
Tần số (f) là số lần dao động trong một đơn vị thời gian.

Biên độ dao động $\mathrm{A}=\sqrt{a_{1}^{2}+b_{1}^{2}}$ là khoảng cách giữa hai thời điểm cực trị liền kề của điểm quan trắc dao động.

Đối với công trình xây dựng các tham số dao động chính cần xác định là:

- Biên độ dao động $(A)$, tần số (f), chu kỳ dao động $(T)$.

- Độ trễ (A $\mathrm{t}$ ) là thời gian từ khi ngoại lực tác động đến khi công trình dao động.

Ngoài ra cần xác định:

- Độ chính xác đo $m_{i}$ cần thiết để xác định được các tham số dao động.

- Thời gian tối thiểu $t_{0}$ cần thiết giữa hai trị đo liền nhau để dãy trị đo được coi là liên tục.

\section{Một số dạng dao động đặc trưng}

2.1. Dịch chuyển đập chắn công trình thủy lợi, thủy điện

Đập chắn của các công trình thủy lợi, thủy điện bị tác động một phía do độ cao mức nước trong hồ chứa ở phía thượng lưu nên bị dịch chuyển. Sự dịch chuyển này có một phần mang tính tuần hoàn, thay đổi theo độ cao mực nước trong hồ ở phía thượng lưu của đập. Chu kỳ dao động phụ thuộc theo lượng mưa ở lưu vực phía 
thượng nguồn và thường có chu kỳ là một năm.

\subsection{Dao động nhà siêu cao tầng}

Nhà siêu cao tầng do tác động của ngoại lực, chủ yếu là gió, làm cho nó bị dao động. Các dao động này có biên độ phụ thuộc vào cường độ và hướng gió, thường là từ $\mathrm{cm}$ đến $\mathrm{dm}$, thậm chí tới mét [4]. Tần số dao động thường tính theo giây. Dao động trong nhà siêu cao tầng làm ảnh hưởng tới công tác trắc địa đảm bảo thi công, đặc biệt là công tác chuyển trục lên tầng. Mô hình quan trắc xác định tham số dao động nhà siêu cao tầng ở hình 2. Ngoài ra còn các tháp siêu cao, cầu treo kích thước lớn, mức nước ở các cảng biển...cũng cần được xác định các tham số dao động.

\subsection{Dao động của cầu treo, cầu dây văng}

Công trình cầu dây, cầu treo hiện nay có kết cấu phức tạp, khả năng mang tải và vượt nhịp lớn. Do chịu ảnh hưởng của nội lực dưới tác động của tải trọng và môi trường bên ngoài gây lên các của dao động trụ tháp, dầm cầu, cáp dây văng so với trạng thái tĩnh của cầu. Các dao động này thường có biên độ, cường độ từ $\mathrm{cm}$ đến $\mathrm{dm}$. Tần số dao động thường tính theo giây. Việc xác định các tham số dao động là cơ sở để thực hiện các hoạt động kiểm tra và bảo trì công trình cầu.

\section{Xử lý số liệu quan trắc liên tục}

\subsection{Loại sai số thô trong dẫy trị đo}

Phương pháp quan trắc hiệu quả để xác định tham số dao động công trình là đo liên tục. Máy TĐĐT có khả năng tự động đo và lưu số liệu với tần suất tính theo giây. Thiết bị đo GPS có khả năng tự động đo hàng triệu trị đo trong một giờ và kết xuất đến 20 kết quả/giây [6].

Dãy kết quả đo có thời gian giữa hai trị đo ngắn so với chu kỳ dao động, đủ để xác định các tham số cần thiết được xem là dãy trị đo thường xuyên, liên tục.

Trong dãy kết quả đo liên tục, trị đo có sai số vượt quá giới hạn cho phép (là các trị đo có sai số thô) cần loại bỏ.

Thuật toán loại sai số thô phù hợp với dãy trị đo liên tục là "cửa sổ trượt" [1]. Số trị đo liên tục trong mỗi cửa sổ là $\mathrm{k}=2 \div \mathrm{K}$. Giá trị $\mathrm{K}$ phụ thuộc vào số trị đo liên tục trong mỗi chu kỳ và độ chính xác các trị đo. Trong một chu kỳ dao động nếu đo liên tục 30 đến 40 trị đo, giá trị K phù hợp là 4 đến 5 [1].

Ảnh hưởng của các trị đo tới việc xác định các tham số dao động là như nhau, nếu độ chính xác kết quả đo và thời gian giữa các trị đo là bằng nhau. Trường hợp này các trị đo được xem là có trọng số bằng nhau. Sau khi loại sai số thô, dãy trị đo còn lại có những khoảng trống do một hoặc nhóm vài trị đo mang sai số thô đã bị loại tạo ra, dẫn tới thời gian giữa các trị đo trong dãy không bằng nhau. Để tái tạo dãy trị đo cùng trọng số cần bổ sung bằng cách nội suy theo giá trị của 2 trị đo hoặc thay đổi trọng số hai trị đo liền kề. Trong điều kiện cho phép, thì giảm thời gian giữa hai trị đo, đồng nghĩa với tăng số lượng trị đo liên tục trong một chu kỳ, sẽ cho kết quả tốt hơn.

\subsection{Thuật toán xác định tham số dao động công trình thông qua một dãy trị đo}

Trong hình 1 , cạnh $S^{A}$ là dãy trị đo cạnh liên tục khoảng cách giữa điểm đặt máy cố định $\mathrm{M}$ và mốc $\mathrm{A}$ ở vị trí ổn định gần công trình. Cạnh $S_{i}^{1}$ là dãy trị đo liên tục khoảng cách giữa điểm $M$ và điểm 1 ở trên đỉnh nhà siêu cao tầng. (Xem hình 1).

Để xác định các tham số dao động ở điểm 1 , khi đo bằng $T \boxminus Đ T$, cạnh $S^{A}{ }_{i}$ chỉ đo một số trị đo cần thiết. Trình tự tính như sau [5]:

Bước 1. Sau khi loại sai số thô, tính trị trung bình cộng $S_{A}^{T B}$, sai số trung phương 
một trị đo $m_{S A i}$ và sai số trung phương của trị trung bình cộng $m_{A}^{T B}$ theo công thức

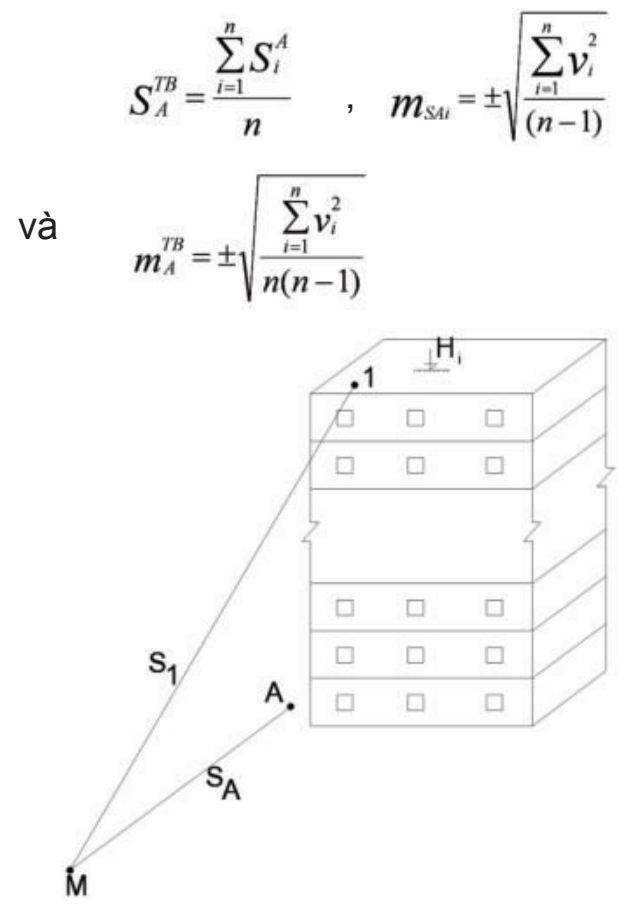

Hình 1: Mô hình quan trắc liên tục bằng $T \boxminus \oplus T$

Bước 2. Tính trị trung bình cộng và sai số của các dãy trị đo liên tục theo cửa sổ trượt.

Mỗi cửa sổ có $\mathrm{k}$ trị đo, $\mathrm{k}=2 \div \mathrm{K}$. Cửa sổ đầu tiên có các trị đo từ 1 đến $\mathrm{K}$. Cửa sổ thứ hai có trị đo từ 2 đến $\mathrm{k}+1$, cửa sổ cuối cùng có các trị đo từ $\mathrm{n}-\mathrm{k}$ đến $\mathrm{n}$. Trong dãy $\mathrm{n}$ trị đo có $\mathrm{j}=\mathrm{n}-\mathrm{K}$ cửa sổ. Mỗi cửa sổ đều tính trị trung bình cộng $S_{j}$ và sai số $m_{S j}$ theo công thức (2), với các trị đo ở cửa sổ thứ j là từ $S_{1}^{T B}$ đến $S_{n-K}^{T B}$

Bước 3. Tính độ lệch trị trung bình cộng giữa hai cửa sổ liền kề thứ $\mathrm{j}$ và $\mathrm{j}+1$ :

$$
\Delta S_{j, j+1}=S_{j+1}^{T B}-S_{j}^{T B}
$$

Trị số $\mathrm{K}$ của cửa sổ trượt được xác định khi tính độ lệch trung bình cho đến khi hội tụ. Trị số $\mathrm{K}$ phù hợp thường biến đổi từ 3 đến 9 . Trị số $K$ phụ thuộc vào độ chính xác mỗi trị đo, số trị đo trong một chu kỳ, biên độ dao động và độ chính xác cần xác định.

Độ lệch có thể hiệu chỉnh ảnh hưởng của điều kiện môi trường thông qua kết quả xử lý dãy trị đo cạnh ổn định $\mathrm{S}_{\mathrm{A}}$ [5] để tăng thêm độ chính xác.

Bước 4. Xác định các tham số dao động

- Chu kỳ dao động T:

Xét dãy độ lệch giữa các cửa sổ trượt, tại vị trí độ lệch đổi dấu là có cực trị. Độ lệch đổi dấu từ dương (+) sang âm (-) là vị trí cực đại; từ âm sang dương có có cực tiểu.

Chu kỳ dao động là tích của số độ lệch giữa hai cực đại hoặc cự tiểu liền kề với thời gian giữa hai trị đo:

$$
\mathrm{t}=\text { No.t }_{\mathrm{o}}
$$

trong đó: No là số độ lệch giữa hai cực đại hoặc hai cực tiểu liền kề. $\mathrm{t}_{\mathrm{o}}$ là thời gian giữa hai trị đo.

- Tần số f là số chu kỳ dao động trong một một đơn vị thời gian

$$
f=1 / T
$$

- Biên độ dao động $A$ là khoảng cách giữa hai cực trị liền kề

Trong đó $\Lambda_{1}$ và $\Lambda_{H+}$ là giá trị của cực trị thứ $\mathrm{j}$ và $\mathrm{j}+1$.

\subsection{Kết quả thực nhiệm}

- Kết quả thực nghiệm 68 trị đo cạnh TĐĐT khoảng cách giữa 2 điểm ổn định mốc $\mathrm{M}$ và mốc $\mathrm{A}$ dưới chân nhà cao tầng là $S_{A}{ }_{A}$ và khoảng cách giữa mốc $M$ và điểm 1 là $S^{i}{ }_{1}$. Điểm 1 ở trên nóc nhà cao tầng bị dao động (hình 1). Biểu đồ 1 là kết quả của hai dãy trị đo liên tục. (Xem biểu đồ 1)

Trị xác suất nhất của 2 cạnh đo là

$$
S_{A}=300,0004 \mathrm{~m} \text { và } S_{1}=301,0128 \mathrm{~m}
$$

Sai số trung phương mỗi trị đo tính theo 
Betsel là

$$
m_{\mathrm{SAi}}= \pm 1,8 \mathrm{~mm} \text { và } m_{\mathrm{S} 1 \mathrm{i}}= \pm 9,2 \mathrm{~mm}
$$

Sai số trung phương trị xác suất nhất là $m_{S A}= \pm 0,2 \mathrm{~mm}$ và $m_{S 1}= \pm 1,1 \mathrm{~mm}$ Biểu đồ 2 là sai số đo cạnh $S_{A}$ và dao động cạnh $\mathrm{S}_{1}$. (Xem biểu đồ 2)

- Thực nghiệm đo GPS cạnh $S_{1}, S_{2}$ và $S_{3}$ nối 3 điểm $A, B$ và $C$ ở các vị trí ổn định; $B a$ cạnh $S_{4}, S_{5}$ và $S_{6}$ là khoảng cách từ 3 điểm ổn định tới điểm $\mathrm{D}$ ở nóc nhà cao tầng, bị dao động (hình 2). (Xem hình 2)

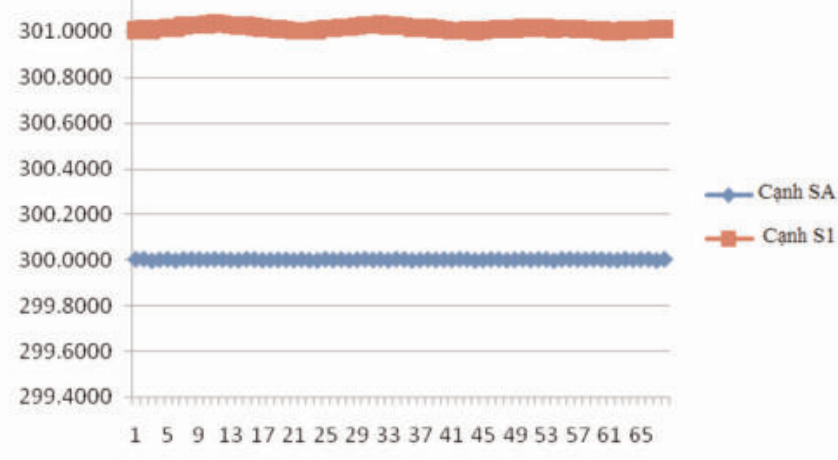

Biểu đồ 1: Kết quả cạnh đo $S_{A}$ và $S_{1}$ BIÉU Đô SAI Só
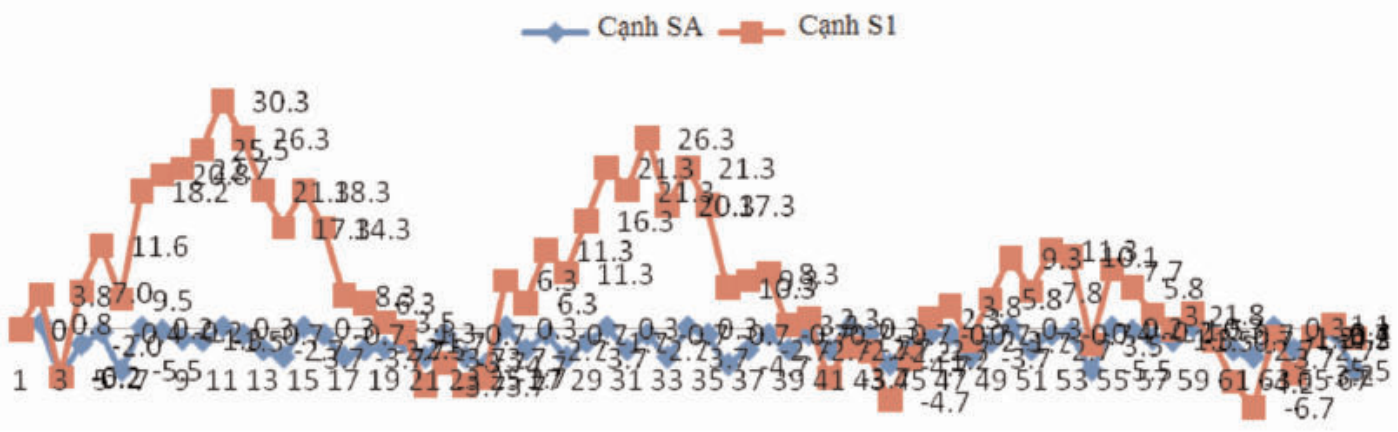

Biểu đồ 2: Sai số cạnh $S_{A}$ và dao động cạnh $S_{1}$

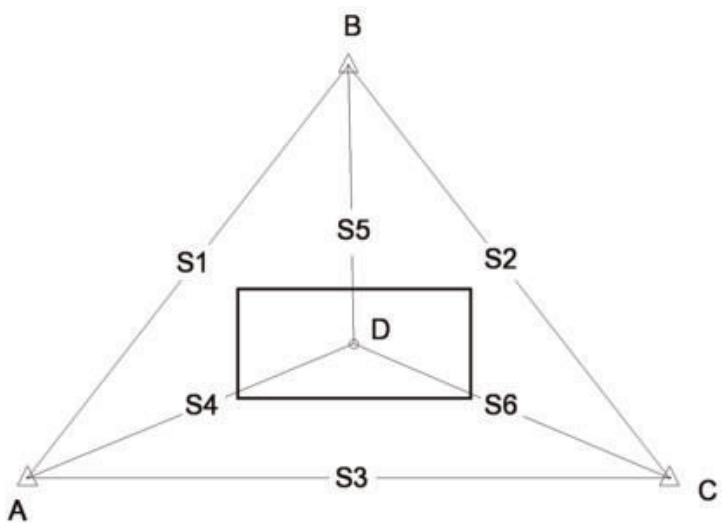

Hình 2: Mô hình quan trắc liên tục bằng GPS 


\section{Trao đổi - Ý kiến}

Xử lý 6 dãy trị đo liên tục và đồng thời với động các cạnh $\mathrm{S}_{4-6}$. (Xem biểu đồ 3) 132 giá trị mối dãy. Kết quả và độ chính xác ở bảng 1. (Xem bảng 1)

Biểu đồ 3 là sai số đo các cạnh $\mathrm{S}_{1-3}$, dao

Biểu đồ 4 là dao động theo 3 hướng $\mathrm{A}, \mathrm{B}$, C và vị trí điểm D. (Xem biểu đồ 4 )

Bảng 1: Kết quả tính 6 cạnh đo liên tục và đồng thời

\begin{tabular}{|c|c|c|c|c|c|c|}
\hline Cạnh & $\mathrm{S}_{1}$ & $\mathrm{~S}_{2}$ & $\mathrm{~S}_{3}$ & $\mathrm{~S}_{4}$ & $\mathrm{~S}_{5}$ & $\mathrm{~S}_{6}$ \\
\hline Trung bình 132 trị đo (m) & 320.1578 & 102.9540 & 319.5309 & 164.0424 & 165.4967 & 200.9666 \\
\hline Sai số trung phương trị đo (mm) & 1.0 & 2.4 & 1.9 & 19.6 & 21.6 & 22.0 \\
\hline Sai số trị trung bình cộng (mm) & 0.1 & 0.2 & 0.2 & 1.7 & 1.9 & 1.9 \\
\hline
\end{tabular}

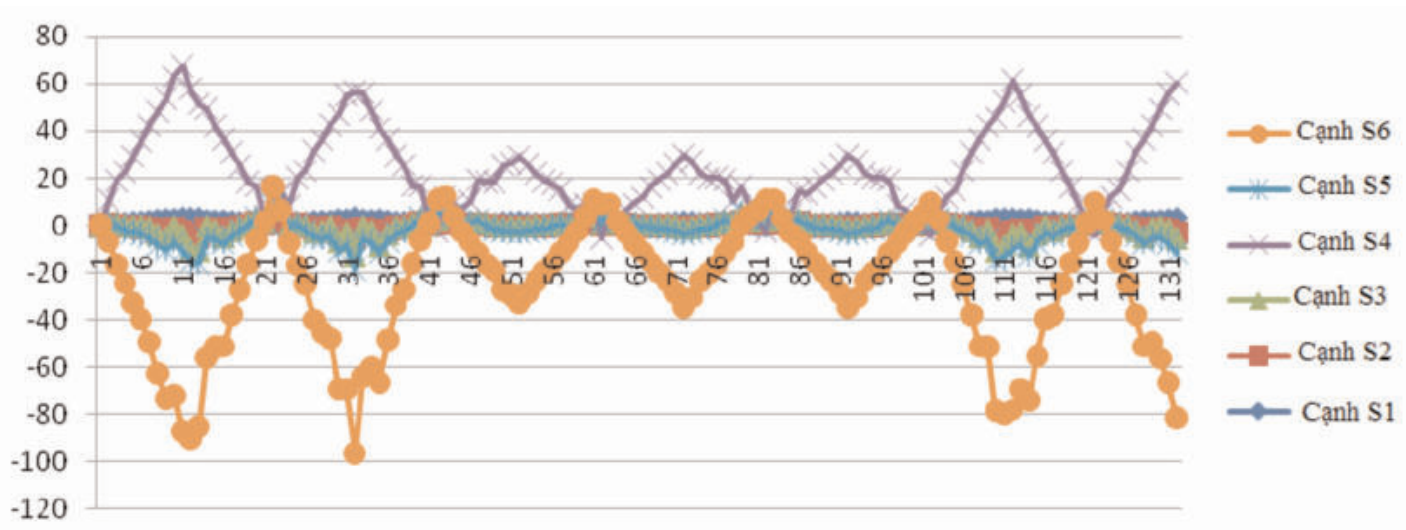

Biểu đồ 3: Dao động điểm D theo 3 hướng

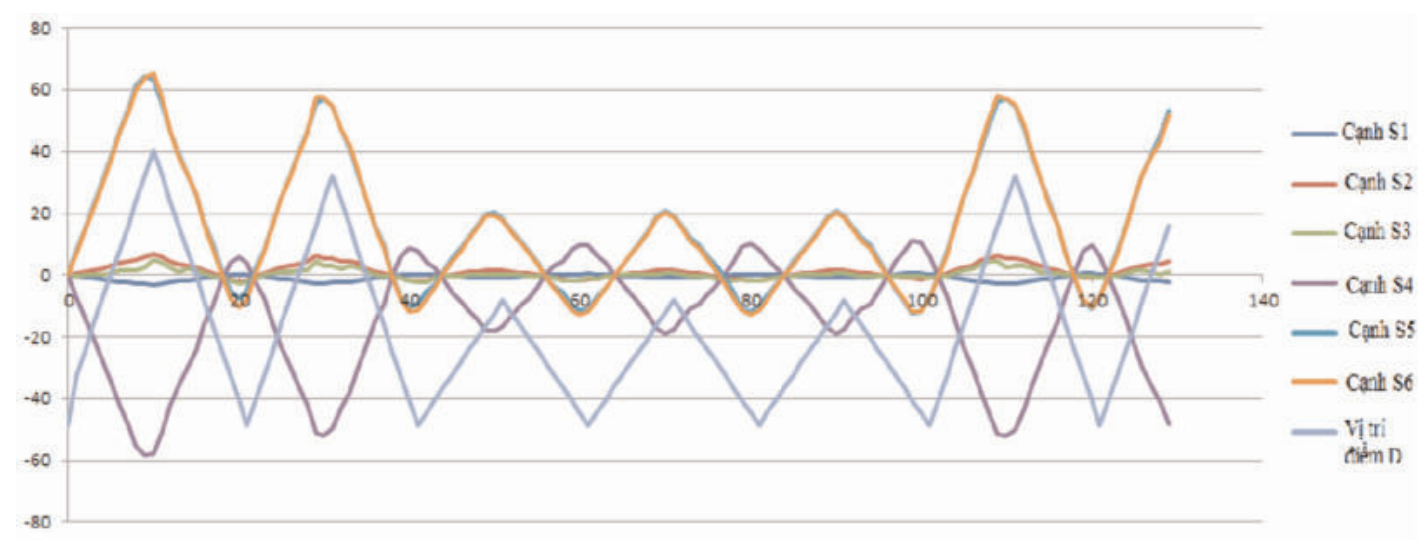

Biểu đồ 4: Dao động theo 3 hướng và vị trí điểm $D$ 
Kết quả xử lý 6 dãy trị đo liên tục, xác định được các tham số dao động điểm quan trắc theo 3 hướng và dao động vị trí điểm.

Khi biên độ dao động tương đương với sai số đo thì việc xác định độ lệch giữa các cửa sổ bị nhiễu, cần có sự can thiệp thủ công. Kết quả khảo sát cho thấy khi biên độ dao động chỉ bằng một nửa sai số đo thì độ lệch hoàn toàn bị nhiễu, không thể xác định được các tham số dao động.

Nếu đo một cạnh, thì chỉ xác định được biên độ dao động theo phương của cạnh quan trắc.

\section{Kết luận và hướng nghiên cứu}

- Dãy trị đo liên tục cho khả năng xác định các tham số dao động của công trình quan trắc.

- Dãy trị đo liên tục cho phép chuyển tọa độ lên nhà siêu cao tầng, nếu xử lý theo qui trình phù hợp thì vị trí tương hỗ giữa các trục không bị ảnh hưởng của dao động.

- Hướng nghiên cứu tiếp theo là hoàn chỉnh phần mềm xử lý số liệu quan trắc liên tục xác định ảnh hưởng của dao động. $\bigcirc$

\section{Tài liệu tham khảo}

\section{Summary}

\section{Method of processing continuous monitoring data indentified oscillation parame- ters works}

Assoc. Prof. Dr. Vu Thang, MSc. Bui Duy Quynh, MSc. Vu Thai Ha

\section{National University of Civil Engineering}

This paper presents results of research into the continuous measurement model and process measurement data to determine the oscillation parameters constructions. When using the modern equipment capable of continuous automatic measurement high accuracy as GPS, total station. $O$

Ngày nhận bài: 29/7/2014.
[1]. Vũ Thặng. Bài giảng Cơ sở Trắc địa

[2]. Nguyễn Quang Thắng. Báo cáo đề tài cấp bộ B2003-36-53. Qui trình công tác trắc địa xây dựng công trình có chiều cao lớn, Hà Nội 2004.

[3]. TCXDVN 4453-1995. Kết cấu bê tông và bê tông cốt thép toàn khối. Qui phạm thi công và nghiệm thu - Hà Nội 1995.

[4]. Huang Sheng Xiang, Liu Jing Nam, Evaluating the precision of GPS positioning for short baseline using long-term data. Wuhan Technical University of Surveying and Mapping, Wuhan 430079, China, 2001.

[5]. Vũ Thặng. Trắc địa xây dựng thực hành. NXB xây dựng, Hà Nội, 2002.

[6]. Công ty cổ phần thiết bị SISC Việt Nam. Các thiết bị trắc địa và địa không gian của Leica. 2010.

[7]. Trần Khánh, Nguyễn Quang Phúc. Quan trắc chuyển dịch và biến dạng công trình. NXB Giao thông vận tải, 2010.

[8]. US. Army Corps of engineers. Structural Deformation Surveying, 2002. 0 công trình, ĐHXD, Hà Nội 2014. 